\title{
H. DJELLOUT
}

\section{A. GUILLIN}

\section{Large and moderate deviations for moving average processes}

Annales de la faculté des sciences de Toulouse $6^{e}$ série, tome 10, $\mathrm{n}^{\mathrm{o}} 1$ (2001), p. 23-31

<http://www.numdam.org/item?id=AFST_2001_6_10_1_23_0>

(C) Université Paul Sabatier, 2001, tous droits réservés.

L'accès aux archives de la revue «Annales de la faculté des sciences de Toulouse » (http://picard.ups-tlse.fr/ annales/) implique l'accord avec les conditions générales d'utilisation (http://www.numdam.org/conditions). Toute utilisation commerciale ou impression systématique est constitutive d'une infraction pénale. Toute copie ou impression de ce fichier doit contenir la présente mention de copyright.

\section{NumDam}

Article numérisé dans le cadre du programme

Numérisation de documents anciens mathématiques

http://www.numdam.org/ 


\title{
Large and moderate deviations for moving average processes ${ }^{(*)}$
}

\author{
H. DJellout AND A. Guillin ${ }^{(1)}$
}

Résumé. - Soit $X_{n}=\sum_{i \in \mathbf{Z}} a_{i+n} \omega_{i}, n \geqslant 1$, le processus à moyenne mobile, $\left(\omega_{i}\right)_{i \in \mathbf{Z}}$ étant une suite de v.a.i.i.d. réelles, et soit $S_{n}=\left(X_{1}+\ldots+\right.$ $\left.X_{n}\right)$. Dans ce papier, nous établissons un principe de grandes déviations et de déviations modérées pour $S_{n} / n$, sous les conditions suivantes : $\omega_{i}$ sont bornées pour tout $i \in \mathbf{Z}$ et $\sum_{n \in \mathbf{Z}} a_{n}^{2}<\infty$.

AbstraCt. - Let $X_{n}=\sum_{i \in \mathbf{Z}} a_{i+n} \omega_{i}, n \geqslant 1$, the moving average process, $\left(\omega_{i}\right)_{i \in \mathbf{Z}}$ i.i.d. real random values, and $S_{n}=\left(X_{1}+\ldots+X_{n}\right)$. In this note, we prove large and moderate deviations principle for $S_{n} / n$, under the boundedness of $\omega_{i}$ and $\sum_{n \in \mathbf{Z}} a_{n}^{2}<\infty$.

\section{Introduction.}

Let $\left\{\omega_{i}, i \in \mathbb{Z}\right\}$ be a doubly infinite sequence of independent and identically distributed square integrable real random variables with $\mathbb{E}\left(\omega_{i}\right)=0$, defined on some probability space $(\Omega, \mathcal{F}, \mathbb{P})$. Let $\left\{a_{n}, n \in \mathbb{Z}\right\}$ be a doubly infinite sequence of real numbers such that $\sum_{i \in \mathbf{Z}} a_{i}^{2}<\infty$.

The moving average process $X_{k}, k \geqslant 1$, is defined by

$$
X_{k}=\sum_{i \in \mathbf{Z}} a_{i+k} \omega_{i},
$$

(*) Reçu le 8 avril 1999, accepté le 8 juin 2001

(1) Laboratoire de Mathématiques Appliquées, Université Blaise Pascal, 24 Avenue des Landais, 63177 Aubière.

email: \{djellout, guillin\}@math.univ-bpclermont.fr 
and let

$$
S_{n}=\sum_{k=1}^{n} X_{k}
$$

Numerous works have been made on the problem of large and moderate deviations of $S_{n} / n$ under the strong condition $\sum_{i \in \mathbf{Z}}\left|a_{i}\right|<\infty$. For example, Burton and Dehling [BD90] have proved a large deviation principle for $\left\{S_{n} / n ; n \geqslant 1\right\}$ with speed $\{n ; n \geqslant 1\}$ and a good rate function depending only on the moment generating function. Their proof, like many of the referenced papers, relies on the powerful Ellis Theorem. The moderate deviations of $\left\{S_{n} / n ; n \geqslant 1\right\}$ are obtained by Jiang and al. [JWR92] under the condition of exponential integrability of $\omega_{0}$. Jiang and al. [JWR95] proved that the upper bound of large deviations for $S_{n}$ in a Banach space $B$ holds if and only if the condition $\mathbb{E}\left(e^{q_{K}(\omega)}\right)<\infty$ is fulfilled for some compact $K$ of $B$, where $q_{K}$ is the Minkowski functional of the set $K$. And the lower bound of large deviations is obtained in [JWR95] without any condition, with a rate function which may not have compact level sets, and which can be different from the rate function of the upper bound.

Remember also the famous work of Donsker and Varadhan [DV85], on large deviations of level-3 for stationary Gaussian processes, under moving average form, which has motivated our study.

In this note, we prove a large deviation principle and a moderate deviation principle for moving average processes, substituting the absolute convergence condition by the continuity of $g(\theta)=\sum_{n \in \mathbf{Z}} a_{n} e^{i n \theta}$ at 0 , a well known condition for the Central Limit Theorem of $\left\{S_{n} / \sqrt{n}\right\}$, see ([HH80], Corollary 5.2. pp 135). But we need the boundedness of $\omega_{i}$ instead of the exponential integrability in the works cited above.

\section{A large deviation principle for the moving average processes.}

About the language of large deviations, see Dembo and Zeitouni [DZ93], Deuschel and Stroock [DS89]. The main result of this paper is

THEOREM 2.1. - Let $\left(\omega_{i}\right)_{i \in \mathbf{Z}}$ be a family of $\mathbb{P}$-i.i.d. real valued random variables. Suppose the following conditions

(H1) $\sum_{i \in \mathbf{Z}} a_{i}^{2}<\infty, \mathbb{E}\left(\omega_{i}\right)=0, \mathbb{E}\left(\omega_{i}^{2}\right)=1$ and $\left|\omega_{i}\right| \leqslant C$ for all $i \in \mathbb{Z}$. 
(H2) The function $g$ given by $|g(\theta)|^{2}:=\left|\sum_{n} a_{n} e^{i n \theta}\right|^{2}=f(\theta)$ (the spectral density function of $X_{k}$ ) is continuous at 0 and belongs to $\mathrm{L}^{2}([-\pi, \pi], d \theta)$.

Then $\mathbb{P}\left(\frac{S_{n}}{n} \in \cdot\right)$ satisfies a large deviation principle with speed $n$ and the good rate function I given by

$$
I(x)=\Lambda^{*}\left(\frac{x}{g(0)}\right), \forall x \in \mathbb{R},
$$

where $\Lambda^{*}$ is the Fenchel-Legendre transform of the logarithmic moment generating function $\Lambda(\lambda):=\log \mathbb{E} e^{\lambda \omega_{0}}$ of the common law of $\omega_{i}, i \in \mathbb{Z}$.

Remark 2.i. - Except the boundedness of $\omega_{i}$, the assumption (H1) is minimal to define $X_{k}$.

Remark 2.ii. - Condition (H2) is the usual condition for the Central Limit Theorem for $S_{n}$, see [HH80]. Notice also that it is much weaker than the condition $f(\theta) \in \mathrm{C}([-\pi, \pi])$ used in the pioneering work of DonskerVaradhan [DV85] for the level-3 large deviations of stationary gaussian processes.

To prove Theorem 2.1 we need the following concentration inequality for $S_{n}$, which is a translation of the well known Hoeffding inequality [Hoe63] in our context.

LEMMA 2.2. - Under condition (H1), we have

$$
\mathbb{P}\left(\left|S_{n}\right| \geqslant t\right) \leqslant 2 e^{-\frac{t^{2}}{2 C^{2} \mathbf{E}\left(S_{n}^{2}\right)}} .
$$

Proof of Lemma 2.2. - By Hoeffding inequality [Hoe63] (or more exactly its proof), applied to

$$
\tilde{S}_{n}^{K}=\sum_{|i| \leqslant K} \tilde{X}_{i}^{n}
$$

where $\tilde{X}_{i}^{n}=\sum_{k=1}^{n} a_{i+k} \omega_{i}$, we have for all $\lambda \geqslant 0$

$$
\begin{aligned}
\mathbb{E}\left(e^{\lambda \tilde{S}_{n}^{K}}\right) & \leqslant \exp \left(\frac{\lambda^{2} C^{2}}{2} \sum_{|i| \leqslant K}\left(\sum_{k=1}^{n} a_{i+k}\right)^{2}\right) \\
& =\exp \left(\frac{\lambda^{2} C^{2}}{2} \mathbb{E}\left(\tilde{S}_{n}^{K}\right)^{2}\right) .
\end{aligned}
$$


Letting $K \rightarrow \infty, \tilde{S}_{n}^{K} \longrightarrow S_{n}$ in $L^{2}(\mathbb{P})$ and we get by Fatou's lemma:

$$
\mathbb{E}\left(e^{\lambda S_{n}}\right) \leqslant e^{\frac{\lambda^{2} C^{2}}{2} \mathbf{E} S_{n}^{2}} \text {. }
$$

Then by Markov inequality, we have that for all $t>0$

$$
\mathbb{P}\left(S_{n} \geqslant t\right) \leqslant e^{-t \lambda+\frac{\lambda^{2} C^{2}}{2} \mathbf{E} S_{n}^{2}}, \forall \lambda \geqslant 0,
$$

and optimizing in $\lambda$, we obtain

$$
\forall t \geqslant 0, \quad \mathbb{P}\left(S_{n} \geqslant t\right) \leqslant e^{-\frac{t^{2}}{2 C^{2} \mathbf{E} S_{n}^{2}}} .
$$

We have obviously the same inequality for $-S_{n}$. Thus (2.2) follows.

Remark 2.iii. - Inequality (2.2) can be proved by means of logarithmic Sobolev inequality for convex functions [Led96] (with less better constant), in a way which is also valid for $\left|S_{n}\right|$ and thus give an alternate way to establish Step 2 in the following proof.

Proof of Theorem 2.1 : we separate its proof into three steps.

Step 1. Let $S_{n}^{K}=\sum_{k=1}^{n} X_{k}^{K}$ where we have for some fixed $K$ in $\mathbb{N}$

$$
X_{k}^{K}=\sum_{|j|<K} a_{j+k}\left(1-\frac{|j|}{K}\right) \omega_{j} .
$$

Then $\mathbf{P}\left(\frac{S_{n}^{K}}{n} \in \cdot\right)$ satisfies the large deviation principle with speed $n$ and some good rate function $I^{K}$, by Sanov's theorem and the contraction principle, or using results of [BD90] which give $I^{K}$ with the same notations as in Theorem 2.1:

$$
I^{K}(x)=\Lambda^{*}\left(\frac{x}{\sum_{|j|<K} a_{j}\left(1-\frac{|j|}{K}\right)}\right) .
$$

Step 2. We show that for all $\delta>0$

$$
\begin{aligned}
\limsup _{K \rightarrow \infty} \limsup _{n \rightarrow \infty} \frac{1}{n} \log \mathbb{P} & \left(\left|\frac{S_{n}}{n}-\frac{S_{n}^{K}}{n}\right|>\delta\right)=-\infty . \\
- & 26-
\end{aligned}
$$


By our hypothesis, we can apply Hoeffding inequality for $S_{n}-S_{n}^{K}$, and noting that $\mathbb{E}\left(S_{n}-S_{n}^{K}\right)=0$ we get by lemma 2.2

$$
\frac{1}{n} \log \mathbb{P}\left(\frac{1}{n}\left|S_{n}-S_{n}^{K}\right|>\delta\right) \leqslant \frac{\log 2}{n}-\frac{n \delta^{2}}{2 C^{2} \mathbb{E}\left(\left(S_{n}-S_{n}^{K}\right)^{2}\right)}
$$

We have now to control the right term of this inequality. Let $f_{K}$ denote the spectral density of $X_{n}^{K}$, i.e. $f_{K}(\theta)=\left|g_{K}(\theta)\right|^{2}:=\left|\sum_{|j|<K} a_{j}\left(1-\frac{|j|}{K}\right) e^{i j \theta}\right|^{2}$.

Let introduce Fejer's kernel $F_{K}$

$$
F_{K}(\theta)=\frac{1}{2 \pi K}\left(\frac{\sin \frac{K}{2} \theta}{\sin \frac{1}{2} \theta}\right)^{2}
$$

An obvious property is that $\int_{-\pi}^{\pi} F_{K}(\theta) d \theta=1$. Moreover, we have $g_{K}=$ $F_{K} * g$, where "*" denotes the usual convolution product. By the well known theorem ([IL71], Theorem 18.2.1. pp322), we have

$$
\frac{\mathbb{E}\left(\left(S_{n}-S_{n}^{K}\right)^{2}\right)}{n}=2 \pi\left[\left|g-g_{K}\right|^{2} * F_{n}\right](0) \text {. }
$$

For any $\varepsilon>0$, let $[-\delta, \delta]$ be such that $|g(\theta)-g(0)|<\frac{\varepsilon}{2}$ for $|\theta|<\delta$. For $|\theta|<\frac{\delta}{2}$

$$
\begin{aligned}
\left|g_{K}(\theta)-g(\theta)\right| & =\left|g * F_{K}(\theta)-g(\theta)\right| \\
& =\left|\int_{-\pi}^{\pi} F_{K}\left(\theta^{\prime}\right) g\left(\theta-\theta^{\prime}\right) d \theta^{\prime}-g(\theta)\right| \\
& \leqslant\left|\int_{\left|\theta^{\prime}\right|<\delta / 2} F_{K}\left(\theta^{\prime}\right)\left(g\left(\theta-\theta^{\prime}\right)-g(\theta)\right) d \theta^{\prime}\right| \\
& \quad+\left|\int_{\left|\theta^{\prime}\right| \geqslant \delta / 2} F_{K}\left(\theta^{\prime}\right)\left(g\left(\theta-\theta^{\prime}\right)-g(\theta)\right) d \theta^{\prime}\right| \\
& \leqslant \varepsilon \int_{\left|\theta^{\prime}\right|<\delta / 2} F_{K}\left(\theta^{\prime}\right) d \theta^{\prime}+C(K, \delta) 2 \pi\left(\|g\|_{L^{2}\left(d \theta^{\prime}\right)}+|g(\theta)|\right) \\
& \leqslant \varepsilon \int_{\left|\theta^{\prime}\right|<\delta / 2} F_{K}\left(\theta^{\prime}\right) d \theta^{\prime}+C(K, \delta) 2 \pi\left(\|g\|_{L^{2}\left(d \theta^{\prime}\right)}+|g(0)|+\frac{\varepsilon}{2}\right)
\end{aligned}
$$

where $C(K, \delta)=\sup _{\left|\theta^{\prime}\right| \geqslant \delta / 2}\left|F_{K}\left(\theta^{\prime}\right)\right| \leqslant\left(2 \pi K \sin ^{2}(\delta / 4)\right)^{-1} \rightarrow 0$ as $K \rightarrow \infty$. We can now control the right hand side of $(2.4)$

$$
\limsup _{K \rightarrow \infty}\left[\left|g-g_{K}\right|^{2} * F_{n}\right](0)=\limsup _{K \rightarrow \infty} \int_{-\pi}^{\pi}\left|g-g_{K}\right|^{2}(\theta) F_{n}(\theta) d \theta
$$




$$
\begin{aligned}
& \leqslant \limsup _{K \rightarrow \infty}\left(\sup _{|\theta|<\delta / 2}\left|g-g_{K}\right|^{2}(\theta)+C(n, \delta) \int_{\left|\theta^{\prime}\right| \geqslant \delta / 2}\left|g-g_{K}\right|^{2}(\theta) d \theta\right) \\
& \leqslant \varepsilon .
\end{aligned}
$$

We further conclude that

$$
\limsup _{K \rightarrow \infty} \limsup _{n \rightarrow \infty} \frac{\mathbb{E}\left(\left(S_{n}-S_{n}^{K}\right)^{2}\right)}{n}=0 .
$$

We then deduce (2.3). Applying the approximation lemma ([DZ93], Th. 4.2.16.), we obtain that $S_{n}$ satisfies the large deviations principle with speed $n$ and the rate function

$$
\tilde{I}(x):=\sup _{\delta>0} \liminf _{K \rightarrow \infty} \inf _{y \in B(x, \delta)} I^{K}(y)=\sup _{\delta>0} \limsup _{K \rightarrow \infty} \inf _{y \in B(x, \delta)} I^{K}(y)
$$

where $B(x, \delta)$ is the ball of radius $\delta$ centered at $x$.

Step 3. It remains to show that $\tilde{I}(x)=I(x)$, where $I$ is given by (2.1). We will first prove that $\tilde{I}(x) \leqslant I(x)$. Assume that $I(x)<\infty$ (trivial otherwise). This inequality is obvious for $x=0\left(\right.$ as $\left.I^{K}(0)=0\right)$. Now for $x \neq 0$, the finiteness of $I(x)$ implies that $g(0) \neq 0$. For each $\delta \geqslant 0$, we have by the convergence of $g_{K}(0)$ to $g(0)$ that $y g(0) \in B(x, \delta)$ implies $y g_{K}(0) \in B(x, 2 \delta)$ for sufficiently large $K$, so that we have

$$
\inf _{z \in B(x, \delta)}\left\{\Lambda^{*}(y), y g(0)=z\right\} \geqslant \inf _{z \in B(x, 2 \delta)}\left\{\Lambda^{*}(y), y g_{K}(0)=z\right\},
$$

which yields $\tilde{I}(x) \leqslant I(x)$.

We now have to prove the converse inequality. Assume at first $g(0) \neq 0$, by the lower semi-continuity of $I$ (inf-compact in reality), we have

$$
\tilde{I}(x)=\sup _{\delta>0} \limsup _{K \rightarrow \infty} \inf _{y \in B(x, \delta)} \Lambda^{*}\left(\frac{y}{g_{K}(0)}\right) \geqslant \liminf _{z \rightarrow \frac{x}{g(0)}} \Lambda^{*}(z) \geqslant \Lambda^{*}\left(\frac{x}{g(0)}\right) .
$$

Now assume $g(0)=0 . \tilde{I}(0) \geqslant I(0)$ (trivial). For $x \neq 0$,

$$
\tilde{I}(x)=\sup _{\delta>0} \limsup _{K \rightarrow \infty} \inf _{y \in B(x, \delta)} \Lambda^{*}\left(\frac{y}{g_{K}(0)}\right) \geqslant \liminf _{|z| \rightarrow+\infty} \Lambda^{*}(z)=+\infty=I(x) .
$$

So we have that $\tilde{I}(x)=I(x)$, which ends the proof of theorem 2.1.

Remarks. - Under the boundedness " $\left|\omega_{i}\right| \leqslant C$ " and the strong condition $\sum_{i \in \mathbf{Z}}\left|a_{i}\right|<\infty$, the level-3 large deviations principle for $\left(X_{n}\right)_{n \in \mathbf{Z}}$ holds. 
Indeed, assume without loss of generality that $\left(\omega_{i}\right)$ is the coordinates system on the product space $\Omega_{C}=[-C, C]^{\mathbf{z}}$ equipped with the product measure $\mathbb{P}=\mu^{\otimes \mathbf{Z}}$, where $\mu$ is the common law of $\omega_{i}$. Let $\theta_{k}$ be the shift operator acting on $\Omega_{C}$, defined by $\left(\theta_{k} \omega\right)_{l}=\omega_{l-k}, \forall k, l \in \mathbb{Z}$, and let $E_{n}$ be the empirical process of the i.i.d. sequence, defined on the space $\mathcal{P}\left(\Omega_{C}\right)$ of all probability measures on $\Omega_{C}$ :

$$
E_{n}=\frac{1}{n} \sum_{k=1}^{n} \delta_{\theta_{k} \omega} .
$$

By the results of Donsker-Vardahan [DV85], $E_{n}$ satisfies a level-3 large deviation principle on $\mathcal{P}\left(\Omega_{C}\right)$ equipped with the weak convergence topology, with speed $n$ and the good rate function given by the Donsker-Varadhan level-3 entropy $H(Q)$, see [DV85] for some details on $H(Q)$. Let $\phi$ be the map given by

$$
\begin{aligned}
\phi: \quad & \rightarrow \mathbb{R}^{\mathbf{Z}} \\
\omega & \rightarrow\left(\sum_{i \in \mathbf{Z}} a_{i+k} \omega_{i}\right)_{k \in \mathbf{Z}} .
\end{aligned}
$$

By the absolute summability $\sum_{i \in \mathbf{Z}}\left|a_{i}\right|<+\infty, \phi$ is continuous from $\Omega_{C}$ to $\mathbf{R}^{\mathbf{Z}}$ both equipped with product topology. Let $\mathcal{P}\left(\mathbf{R}^{\mathbf{Z}}\right)$ be the space of all probability measures on $\mathbb{R}^{\mathbf{Z}}$ equipped with the weak convergence topology. Define on $\mathcal{P}\left(\mathbb{R}^{\mathbf{Z}}\right)$ the empirical measure

$$
R_{n}=\frac{1}{n} \sum_{k=1}^{n} \delta_{X_{\cdot+k}} .
$$

We obviously have $R_{n}=E_{n} \circ \phi^{-1}$. By the contraction principle, we conclude that $R_{n}$ satisfies a level-3 large deviation principle on $\mathcal{P}\left(\mathbb{R}^{\mathbf{Z}}\right)$ with speed $n$ and the good rate function $I(Q)=\inf \left\{H(\tilde{Q}) ; Q=\tilde{Q} \circ \phi^{-1}\right\}$.

\section{Moderate deviations.}

We are now studying moderate deviations for $S_{n}$ in the same way as we have proved large deviations in the preceding section, we keep the same conditions on $a_{i}$ and $\omega_{i}$. To this purpose, let $\left(b_{n}\right)_{n \geqslant 1}$ be a sequence of positive numbers such that

$$
b_{n} \rightarrow+\infty \quad, \frac{b_{n}}{\sqrt{n}} \rightarrow 0
$$


H. Djellout and A. Guillin

THEOREM 3.1. - Under the conditions (H1) and (H2), $\mathbb{P}\left(\frac{S_{n}}{b_{n} \sqrt{n}} \in \cdot\right)$ satisfies a large deviation principle with the speed $b_{n}^{2}$ and the good rate function $I_{M}$ given by

$$
\forall x \in \mathbb{R}, \quad I_{M}(x)=\frac{x^{2}}{2 f(0)} .
$$

Proof. - We separate its proof into two steps.

Step 1. Let $S_{n}^{K}=\sum_{k=1}^{n} X_{k}^{K}$ as before.

Then, by [JWR92], $\mathbb{P}\left(\frac{S_{n}^{K}}{b_{n} \sqrt{n}} \in \cdot\right)$ satisfies a large deviation principle with speed $b_{n}^{2}$ and the good rate function $I_{M}^{K}$ given by

$$
I_{M}^{K}(x)=\frac{x^{2}}{2\left(\sum_{|j|<K} a_{j}\left(1-\frac{|j|}{K}\right)\right)^{2}} .
$$

Step 2. Since $S_{n}-S_{n}^{K}$ satisfies assumptions of lemma 2.2, we apply the concentration inequality (2.2) to $\frac{S_{n}-S_{n}^{K}}{b_{n} \sqrt{n}}: \forall t \geqslant 0$,

$$
\frac{1}{b_{n}^{2}} \log \mathbb{P}\left(\left|\frac{S_{n}-S_{n}^{K}}{b_{n} \sqrt{n}}\right| \geqslant t\right) \leqslant-\frac{n t^{2}}{2 C^{2} \mathbb{E}\left(\left(S_{n}-S_{n}^{K}\right)^{2}\right)}+\frac{\log 2}{b_{n}^{2}} .
$$

But by the proof of Theorem 2.1 we have

$$
\limsup _{K \rightarrow \infty} \limsup _{n \rightarrow \infty} \frac{\mathbb{E}\left(\left(S_{n}-S_{n}^{K}\right)^{2}\right)}{n}=0
$$

where it follows

$$
\limsup _{K \rightarrow \infty} \limsup _{n \rightarrow \infty} \frac{1}{b_{n}^{2}} \log \mathbb{P}\left(\left|\frac{S_{n}-S_{n}^{K}}{b_{n} \sqrt{n}}\right| \geqslant t\right)=-\infty .
$$

According to the approximation lemma ([DZ93], Th. 4.2.16.), we deduce that $S_{n}$ satisfies the moderate deviations principle with speed $b_{n}^{2}$ and the rate function

$$
\tilde{I}_{M}(x):=\sup _{\delta>0} \liminf _{K \rightarrow \infty} \inf _{y \in B(x, \delta)} I_{M}^{K}(y)=\sup _{\delta>0} \limsup _{K \rightarrow \infty} \inf _{y \in B(x, \delta)} I_{M}^{K}(y) .
$$


Large and moderate deviations for moving average processes

The identification of the rate function is done like in Step3 of the proof of theorem 2.1.

Acknowledgment. - The authors thank Pr. Liming Wu for fruitful discussions and advices. We are grateful to the anonymous referee for his valuable comments which improve the presentation of this work.

\section{Bibliography}

[BD90] BURTON (R.M.) and DehLing (H.). - Large deviations for some weakly dependent random processes. Statistics and Probability Letters, 9:397-401, 1990.

[DS89] Deuschel (J.D.) and Stroock (D.W.). - Large deviations. Academic Press, Boston, 1989.

[DV85] DONSKER (M.D.) and VARADHAN (S.R.S.). - Large deviations for stationary gaussian processes. Communications in Mathematical Physics, 97:187-210, 1985.

[DZ93] Dembo (A.) and ZeItouni (O.). - Large deviations techniques and their applications. Jones and Bartlett, Boston, MA, 1993.

[HH80] HALL (P.) and HEYDE (C.C.). - Martingale limit theory and its application. Academic Press, New York, 1980.

[Hoe63] HoEFfDING (W.). - Probability inequalities for sums of bounded random variables. American Statistical Association Journal, pages 13-30, 1963.

[IL71] IBRAGIMOV (I.A.) and LINNIK (Y.V.). - Independent and stationnary sequences of random variables. Wolters-Noordhoff Publishing, 1971.

[JWR92] JiANG (T.), WANG (X.) and RAO (M.B.). - Moderate deviations for some weakly dependent random processes. Statistics and Probability Letters, 15:7176, 1992.

[JWR95] JIANG (T.), WANG (X.) and RAO (M.B.). - Large deviations for moving average processes. Stochastic Processes and Their Applications, 59:309-320, 1995.

[Led96] LEDoux (M.). - On Talagrand's deviation inequalities for product measures. ESAIM: Probability and Statistics, 1:63-87, 1996.

[Led99] LEDoux (M.). - Concentration of measure and logarithmic Sobolev inequalities. Séminaire de Probab. XXXIII, LNM Springer, 1709:120-216, 1999. 